\title{
TANA: efficient approach for predicting protein functions by transferring annotation via alignment networks
}

Warith Eddine DJEDDI ( $\square$ warithsa5@gmail.com )

Universite de Tunis El Manar https://orcid.org/0000-0001-5751-2371

Sadok BEN YAHIA

Tallinn University of technology

Engelbert MEPHU NGUIFO

Universite Clermont Auvergne - Campus des Cezeaux

Methodology article

Keywords: PPI; networks; alignment; function prediction; neighborhood; gene ontology; computational assessment of function annotation

Posted Date: June 11th, 2020

DOI: https://doi.org/10.21203/rs.2.21071/v3

License: (c) (1) This work is licensed under a Creative Commons Attribution 4.0 International License. Read Full License 


\title{
TANA: efficient approach for predicting protein functions by transferring annotation via alignment networks
}

\author{
Warith Eddine DJEDDI ${ }^{*}$, Sadok BEN YAHIA ${ }^{1,2}$ and Engelbert MEPHU NGUIFO ${ }^{3}$
}

${ }^{*}$ Correspondence:
warithsa5@gmail.com
${ }^{1}$ University of Tunis EI Manar,
Faculty of Sciences of Tunis,
LR11ES14, Capmus
Universitaire, 2092 Tunis, Tunisia
Full list of author information is
available at the end of the article

${ }^{*}$ Correspondence: warithsa5@gmail.com Faculty of Sciences of Tunis, LR11ES14, Capmus Full list of author information is available at the end of the article

\begin{abstract}
Background: One of the challenges of the post-genomic era is to provide accurate function annotations for orphan and unannotated protein sequences. With the recent availability of huge PPIs networks for many model species, the computational methods revealed a great requirement to elucidate protein function based on many strategies. In this respect, most computational approaches integrate diverse kinds of functional interactions to unveil protein functions by transferring annotations across different species by relying on similar sequence, structure 2D/3D, amino acid patterns or phylogenetic profiles.

Results: In this work, we introduce a new approach, called TANA, for inferring protein functions. The main originality of the introduced approach stands on the function prediction for the unannotated protein by transferring annotation via a network alignment as well as from the direct interaction neighborhood within their PPI networks. Doing so, we are able to discover the functions of proteins that could not to be easily described by sequence homology. We assess the performance of our approach using the standard metrics established by the CAFA challenge and highlight a sharp significant improvement over other competitive methods, in particular for predicting molecular functions.
\end{abstract}

Conclusions: This research is one of the first attempts that combine sequence and networks-multiple-alignment-based function prediction approaches. We have been able to assess the accuracy of the prediction using pairwise and multiple alignment of the PPI networks for the compared species. Therefore, we recommend using different strategies (i.e pairwise, multiple, with/without neighborhood networks) especially in situations where the functions of the protein are not known beforehand.

Keywords: PPI; networks; alignment; function prediction; neighborhood; gene ontology; computational assessment of function annotation

\section{Background}

The past decade has witnessed an increasing in genomic and proteomic data, leading to a large variety of sequenced proteins. A fundamental challenge is the interpretation of this overwhelming data in order to elucidate more accurate protein functions. The manual annotation of protein function is a daunting task which paves the way to the emergence of successful computational predictive methods. The latter have been applied starting from incorporating gene expression patterns [1], phylogenetic profiles [2, 3], protein sequences $[4,5]$, protein structures [6, 7], and protein interactions [8]. A wealthy number of computational approaches for predicting function from networks have been proposed can be 
organized into two major classes: $(i)$ those using a direct network-context: to wit the direct annotation of proteins infer functions based on its connections in the network; (ii) those assisted by a prediction module. The latter first identifies clusters, or modules, of related proteins and then annotates each protein based on the known functions of its members [9].

There are many strategies that have been developed to assign a function to an unknown protein:

- Gene expression pattern: protein function prediction by analyzing gene expression patterns [10].

- Phylogenetic profile: analyzing phylogenetic profile, i.e. evolutionary history of proteins [11].

- Protein sequence: protein function prediction using protein sequence, sequence similarity measures, homologies are primarily used $[12,13]$.

- Protein structure: the function prediction using protein structure, by using some approaches to analyze the secondary $[14,15,16]$ and tertiary structures $[17,6]$ of proteins.

- Protein-protein interactions (PPIs): protein function prediction using proteinprotein interactions $[18,19,20,21,22,23,24,25]$ can be deduced from the interaction of the neighborhood. Chua et al. [26] underscored the useful strategies using the PPIs as a complementary approach to sequence homology by specifying the maximum additional coverage for the PPIs.

- Network alignment: the function prediction using annotation transfers via network alignment by conferring the annotations of a protein in an aligned cluster to the unannotated member of the identical cluster [27, 28]. However, a thorough analysis highlights that such automated transfers may not always be adequate to feed correct function predictions. Indeed, integrating the results of global alignment in order to predict function, gives more trusty predictions [29].

All of these factors have provided an increase in a varied number of automated approaches based on a number of features (i.e. Direct or module assisted approaches) [9]. The FFPRed 3 [30] is an approach for protein function prediction based on the scanning of the input protein sequences accross an array of SVMs considering the tie between protein function and alternative motifs. GoFDR [31] is an alignment-based method for protein function prediction from the query sequence-based MSA produced by BLAST or PSIBLAST search. After that, it induces the FDRs for a target GO term and builds up a PSSM for the FDRs. Finally, it scores the protein target using the PSSM, and tuning the raw score into probability.

DeepText2GO [32] applies a 'consensus' strategies to integrating text-based with sequence-based classifiers. Furthermore, the approach uses a myriad of protein information, such as sequence homology, families, citations, domains, and motifs. In the same trend, GOLabeler [33] integrates five component classifiers, trained from different features, such as GO term frequency, sequence alignment, amino acid trigram, domains and motifs, and biophysical properties, in the LTR machine learning framework. Meanwhile, the authors developed the NetGO web server [34], which incorporates protein-protein network information from the STRING database into the GOLabeler framework (i.e. LTR). NetGO can use a network information to annotate a protein at a large-scale level by using the massive network information of all species in STRING.

DeepGO [35] is an approach for predicting protein functions from protein sequences and PPI networks. It applies a neural networks to learn sequence and PPI network elements and 
hierarchically classifies it with GO classes. INGA [36] is also an other approach to predict protein functions from a combination of "sequence similarity" (by using BLAST [12]), "domain architecture searches" (by using PFAM [37]) and "PPI networks data" (by using STRING [38]) into a consensus prediction for each of the three GO sub-ontologies, MF, BP and CC. INGA 2.0 [39] is a new version of the INGA approach for protein function prediction. While the latter makes use homology, domain architecture, PPI interaction networks and information from the 'dark proteome', in order to produce a consensus for the prediction process. INGA 2.0 transfers GO terms from proteins with the same domain architecture. Moreover, after filtering out of the direct interactions from the STRING database, the transfer is performed with a probability illustrating their enrichment in comparison to the STRING database.

The DeepGOPLUS approach [40] is an extended and improved version of the DeepGO in order to mitigate the downsides of this latter. To do so, DeepGOPLUS combines neural network predictions with methods based on sequence similarity to capture orthology and, indirectly, some interaction information. The authors improved the previous version by replacing the amino acid trigram embedding layer with a parameter-free one-hot encoding. Furthermore, the novel approach have applied a flat classification layer rather than performing the hierarchical classification in DeepGO.

Additionally, there are some additional approaches, which have been applied to predict protein function based on shared binding residues [41], the guilty by-association rules [39], e.g. the neighbor-counting method [42] and the Chi-square method [43]. Worthy of mention, a thorough review on methods in automated protein function prediction is provided in [44]. Although many computational approaches have been developed in recent years to predict protein function, for the $\mathrm{CAFA}^{[1]}$, most of these traditional algorithms do not use the PPI alignment to predict the function for unknown proteins.

In this paper, we introduce TANA a new approach to predict protein function by exploiting PPI networks, sequence similarity and functional similarity. The new strategy predicts the functional annotation of proteins through the comparison of multiple PPIs networks from different species.

The TANA approach is inspired by our former MAPPIN approach [45]. Nevertheless, thorough improvements have been introduced in order to incorporate the GNA results as an entry to the prediction process. Firstly, the semantic similarity between each compared proteins is computed during the alignment process by only excluding the GOA with evidence code IEA. Secondly, we modified the output identifier of the proteins belonging to each cluster (i.e. the sets of the proteins resulted from the alignment process) from the UniProtKB identifier to the CAFA target identifier.

MAPPIN is a global alignment of multiple PPIs networks, which relies on combining sequence similarity, network topology similarity and functional similarity in order to align $k$ multiple networks. The alignment approach uses sequence similarity together with the GOA of proteins to incorporate functional similarity between the proteins. The approach relies on the functional similarities (i.e, SCHLICKER similarity) [46] during the alignment process.

The MAPPIN approach operates in four major steps [45]:

1 Parsing the $k$ PPI networks;

\footnotetext{
${ }^{[1]}$ https://www. biofunctionprediction.org/cafa/
} 
2 Giving a computed weight to each edge in the bipartite graphs using the similarity from the GOA and sequence level for each aligned protein. The GOA database contains a list of associations between UniProtKB identifiers and GO terms;

3 Collecting seed with high similarity scores from the bipartite graphs. Then, each seed is expanded in an iterative fashion by exploring the local neighborhood for each compared protein;

4 Finally, MAPPIN applies a simulated annealing (SA) function in order to find a global alignment between the compared species (i.e. Eukaryotes or Prokaryotes).

By contrast, the TANA approach uses GNA with $k$-networks, by identifying modules of related proteins and then annotate each module based on the most frequent annotations. Our method uses the alignment of the PPI networks from different species to discover functionally similar or conserved protein modules between them. Two major steps are involved:

1 Discover the modules or clusters which are functionally coherent using a largely revised version of the MAPPIN method;

2 Discover the neighbors of a given unannotated protein that share a common functionality, especially by finding densely connected regions in a PPI network. Indeed, this neighborhood may lead to enhance the prediction results by adding the set of proteins interacting with the unannotated protein. The approach relies on the SCHLICKER functionality similarity [46] to compare the GO terms assigned to each neighbor of each unannotated protein (belonging to each discovered aligned nodes) in order to deduce the most frequent annotations. Besides, it is worth noting that this strategy leads to discover a functional module. The latter corresponds to an association of proteins involved in a common biological process or a molecular function and binding among themselves at different times and places.

It is worth mentioning that our approach is the first one that predicts functionality of unannotated proteins from transferring annotations via multiple GNA by integrating the GOA during the alignment process. Indeed, it gives us an advantage to predict useful and accurate function for unknown proteins. Our approach aims to find a multiple global network alignment, with $k$-networks, in order to find out clusters of proteins across the $k$-compared networks, such that these clusters depict a conserved biological function. Here, we explore the possibility of using the GOA, i.e., functional similarity of protein between the compared networks to extract modules that correspond to specific biological processes by increasing the number of conserved interactions.

\section{Results}

Test Datasets

As a dataset for our prediction process, we used :

- The GO released in 2016_05;

- In addition to protein sequences similarity computed from the BLAST, we use PPI networks for multiple species from the STRING database [47] (cf. Table 1);

- The datasets from UniProtKB-GOA released in 2016_05 for the compared species from the CAFA3 challenge. We applied two datasets (i.e. D1 and D2) to align the different species (cf. Table 2), since it gives the best prediction quality, respectively in terms of protein-centric, term-centric and time ratio;

- The protein targets released on November 15th 2017 that had no function annotations at the time of training. It is available for download at https:// biof unctionprediction.org/cafa/; 
- The different alignment strategies (i.e pairwise or multiple alignment) for eukaryotes and prokaryotes species are described respectively in Table 2 and Additional file 1: Table S1.

Experimental Setup

TANA provides different options of protein function prediction in different modes including:

- TANA-1: the prediction process is only based on transferring shared annotation via multiple PPI networks alignment of D1 and D2 (cf., Table 2).

- TANA-2: the prediction process is based on the transfer of annotations by considering the shared GOA and the network neighborhood via multiple PPI network alignments of D1 and D2 (cf., Table 2).

- TANA-3: the prediction process is only based on transferring shared annotations via pairwise PPI networks alignment of D3 and D4, as shown respectively in Additional file 1: Table S1.

- TANA-4: the prediction process is based on the transfer of annotations by considering the shared GOA and the network neighbours via pairwise PPI networks alignments of D3 and D4, as shown respectively in Additional file 1: Table S1.

Evaluation Metrics

The strategy used to evaluate TANA versus its competitors approaches is different from the CAFA3 evaluation. In our strategy, the annotations are propagated using the whole structure of the GO hierarchy [48] by inspecting all types of relationships among GO terms. After that, the parent and child GO terms are dissociated into their individual sub-ontologies (i.e. MFO, BPO and CCO). This strategy is also applied by DeepGOPLUS [40], GOLabeler [33] and DeepText2GO [32]. Using this evaluation, we can conclude that the process of inferring the parent and child terms are over the whole GO. Whereas for the CAFA3 evaluation [49], each single GO term is initially dissociated by their three sub-ontologies. Then, the parent and the child terms are then taken locally into their three respective subontologies.

Therefore, to evaluate the quality of protein function prediction for each algorithm, we consider two scenarios $^{[2]}$ : (i) protein centric; and (ii) term centric.

\section{1) Protein-centric metrics:}

The protein centric maximum F-measure is used in the CAFA3 challenge [50]. Here, we compute the F-measure, for a threshold $t \in[0,1]$, using the average precision for proteins for which we predict at least one term and average recall for all proteins. Then, we select the maximum F-measure value of all thresholds. We compute the $F_{\max }$ measure using the following formulas:

$$
p r_{i}(t)=\frac{\sum_{f} I\left(f \in P_{i}(t) \wedge f \in T_{i}\right)}{\sum_{f} I\left(f \in P_{i}(t)\right)}
$$

${ }^{[2]}$ The python code to evaluate our approach and its competitors is available at http://doi. org/10.5281/zenodo. 3726187 


$$
\begin{aligned}
& r_{i}(t)=\frac{\sum_{f} I\left(f \in P_{i}(t) \wedge f \in T_{i}\right)}{\sum_{f} I\left(f \in T_{i}\right)} \\
& \operatorname{AvgrPr}(t)=\frac{1}{m(t)} \cdot \sum_{i=1}^{m(t)} p r_{i}(t) \\
& \operatorname{AvgrRc}(t)=\frac{1}{n} \cdot \sum_{i=1}^{n} r c_{i}(t) \\
& F_{\text {max }}=\max _{t}\left\{\frac{2 \cdot \operatorname{AvgrPr}(t) \cdot \operatorname{AvgrRc}(t)}{\operatorname{AvgrPr}(t)+\operatorname{AvgrRc}(t)}\right\}
\end{aligned}
$$

In these measures, $f$ stands for GO term, $P_{i}(t)$ denotes the set of terms for a protein $i$ applying a threshold $t$, and $T_{i}$ denotes the corresponding ground-truth set of terms for the protein $i$. Precision is averaged over the proteins with at least one predicted score greater than or equal to $t$ and $m(t)$ is the number of such proteins. The parameter $n$ stands for the number of targets used in such evaluation. Moreover, we have used the remaining uncertainty $(r u)$, misinformation $(m i)$ and the resulting minimum semantic distance $\left(S_{m i n}\right)$ to evaluate the performance of our approaches. The latter metrics are defined as follows:

$$
\begin{aligned}
& r u(t)=\frac{1}{n} \sum_{i=1}^{n} \sum_{c \in T_{i}-P_{i}(t)} I C(c), \\
& m i(t)=\frac{1}{n} \sum_{i=1}^{n} \sum_{c \in P_{i}(t)-T_{i}} I C(c), \\
& S_{\min }=\min _{t}\left\{\sqrt{r u(t)^{2}+m i(t)^{2}}\right\},
\end{aligned}
$$

where $I C(c)$ refers for the information content of the ontology term, which is computed based on the annotation probability of the class $c$ :

$$
\operatorname{IC}(c)=-\log (\operatorname{Pr}(c \mid P(c)))
$$

where $P(c)$ a set of parent classes of the class $c$.

\section{2) Term-centric metrics:}

We have also evaluated our results on the term-centric evaluation. To do so, for each GO term $f$, we compute the AUC for the ROC of a sensitivity (or a recall) for a given false positive rate (or 1-specificity). We compute the sensitivity and the specificity for a functional 
GO term $f$ and threshold $t$ as follows:

$$
\begin{aligned}
& s n_{f}(t)=\frac{\sum_{i} I\left(f \in P_{i}(t) \wedge f \in T_{i}\right)}{\sum_{i} I\left(f \in T_{i}\right)} \\
& \operatorname{sp}_{f}(t)=\frac{\sum_{i} I\left(f \notin P_{i}(t) \wedge f \notin T_{i}\right)}{\sum_{i} I\left(f \notin T_{i}\right)}
\end{aligned}
$$

where $P_{i}(t)$ denoting the set of predicted GO terms for a protein $i$ by applying a threshold $t$, and $T_{i}$ denotes the corresponding ground-truth set of terms for a protein $i$. Then, we define a term-centric $F_{\text {max }}$ measure, where for each GO term $f$ we compute the F-measure using a threshold $t$ and all proteins in our test set. After that, we fix the maximum for all the thresholds.

$$
\begin{aligned}
& p r_{f}(t)=\frac{\sum_{i} I\left(f \in P_{i}(t) \wedge f \in T_{i}\right)}{\sum_{i} I\left(f \in P_{i}(t)\right)} \\
& r c_{f}(t)=\frac{\sum_{i} I\left(f \in P_{i}(t) \wedge f \in T_{i}\right)}{\sum_{i} I\left(f \in T_{i}\right)} \\
& F_{\text {maxf }}=\max _{t}\left\{\frac{2 \cdot p r_{f}(t) \cdot r c_{f}(t)}{p r_{f}(t)+r c_{f}(t)}\right\}
\end{aligned}
$$

Moreover, we compute the global ROC AUC for all predictions scores and Mathews Correlation Coefficient (MCC) for a threshold. Doing so, we compute a maximum proteincentric F-measure. Therefore, the ROC AUC metric is computed by applying the following formulas for a given threshold $t$ :

$$
\begin{aligned}
& A U C=\int_{-\infty}^{n} \operatorname{TPRT}()\left(-F P R T^{\prime}(t)\right) d t \\
& T P R(t)=\frac{T P(t)}{T P(t)+F N(t)}, F P R(T)=\frac{F P(t)}{F P(t)+T N(t)}
\end{aligned}
$$

$T P$ is the cardinality of true positive elements, $F N$ is the cardinality of false negatives, $F P$ is the cardinality of false positive elements and $T N$ is the cardinality of true negatives elements. By doing so, the $M C C$ metric is computed as follows:

$$
M C C=\frac{T P . T N-F P . F N}{\sqrt{(T P+F P)+(T P+F N)+(T N+F P)+(T N+F N)}}
$$

Moreover, we plotted precision-recall curves for each approach for BP, CC and MF ontologies. The AUPR metric is applied in our evaluation in order to disadvantage false positive prediction especially in highly imbalanced data [51]. 


\section{Discussion}

Application to protein function prediction

Table 1 sketches an idea about the number and characteristics of species used by the CAFA3 challenge. The BP, CC and MF respective columns, in Table 2, show the number of proteins that have no experimental annotation in the current ontology category, but having experimental annotations in at least another ontology. We compared our approach, respectively, versus DeepGO, GoFDR, DeepGOPLUS, INGA-Tosatto and orengo-funfams approaches as well as three baselines: DiamondBLAST, DiamondScore and Naive approach, which thoroughly described in [52]. Table 4 depicts the results obtained by the four versions of TANA ${ }^{[3]}$ (i.e. TANA-1, TANA-2, TANA-3 and TANA-4) and its competitors. The GoFDR preliminary prediction file for CAFA3 targets are retrieved from the web service available at http://gofdr.tianlab.cn/ and the full benchmark prediction were not accessible for this approach. Moreover, the INGA-Tosatto prediction files are available at this web link https://inga.bio.unipd.it/db/CAFA3_ INGA-Tosatto_predictions_model1.tar.gz. Adding to that, we thank the authors of the orengo-funfams approach for sharing with us their predictions files. The available evaluation (NK benchmarks /full mode) on CAFA3 challenge, shows that orengo-funfams and the INGA-Tosatto are ranked among the top 10 for CC, BP and MF prediction.

\section{Protein-centric evaluation}

The twelve approaches, as shown in Table 4, produce different results to unannotated proteins for the considered species. Indeed, the TANA-1 algorithm outperforms, in terms of $F_{\text {max }}$, all of its competitors, specifically for predicting MF terms. Whereas, DeepGO, DeepGOPLUS, orengo-funfams, INGA-Tosatto, DiamondBLAST and DiamondScore outperform TANA in BP and CC terms for $F_{\max }, A U C$ and $M C C$ metrics. The influence of mixing the shared gene ontology annotations with network neighborhood is clearly noticeable through the alignment of multiple PPI performed by TANA-1 and TANA-2. Indeed, the accuracy of the prediction of TANA- 1 on the MF category in terms of $F_{\text {max }}$ evaluation is equal to 0.63 , whereas for the TANA-2 was 0.54 (cf. Table 4).

Indeed, it is, respectively, that the orengo-funfams approach performs well for $F_{\max }$ and $S_{\text {min }}$ for MF and BP evaluations under the CAFA3 challenge [49]. The approach ranked 2nd and 4th in $F_{\max }$ for MF and BP, as well as, respectively, 2nd and 3rd in $S_{\min }$ for MF and BP evaluations. Besides, the INGA-Tosatto also performs well for $F_{\max }$ and $S_{\min }$ for $\mathrm{BP}$ and $\mathrm{CC}$ predictions. The approach was ranked, respectively, 2nd and $3 \mathrm{rd}$ in $F_{\text {max }}$ for BP and CC, as well as 2 nd in $S_{\text {min }}$ for BP and CC evaluations [49].

As shown in Table 4 as well as in the Additional file 2: Table S2, predicting the BP terms is more critical process than predicting MF terms. Indeed, BP terms illustrate the relations between proteins, whereas those of MF terms underscore the properties of a protein. Therefore, we can conclude that the feature or property of a given protein is determined by itself, whereas the relations of a protein, with its neighborhood, are not determined by themselves, i.e., relying on other proteins. Thus, applying the sequence of a protein and other features (i.e. the PPIs interaction network to which the unannotated protein belongs) during the prediction process helps us to sharply identify the BP or the $\mathrm{CC}$ for each target protein. Whereas in our case, we align different species that have distinct PPI networks.

${ }^{[3]}$ Details about the prediction results in different modes of TANA are visible at: http://doi. org/10.5281/zenodo. 3718451 
So, it is very arduous to deduce or to predict the BP and CC terms for highly dissimilar proteins.

The protein-centric performance assesses the accuracy of the approaches in assigning functional GO terms to an unannotated protein. Therefore, and as protein-centric evaluation shows (cf. Table 4), our approach flags out good performances in predicting the MF terms to each unannotated proteins. Indeed, our approach does not only rely on the homologs during the alignment process but it also takes the strength of the functional similarity from the GOA for each protein. Moreover, the out performance of TANA over its competitors for the MF prediction is owe to its ability to predict the functionality of unannotated proteins from different species even if the target proteins are not related by an homology sequence.

Figure 2 depicts that TANA-1 outperformed the other approaches in the MF ontology by achieving the highest AUPR in the MF ontology, while DeepGOPLUS outperforms all the approaches in predicting BP and CC terms. In fact, TANA-1 achieved roughly $2 \%$ improvements over orengo-funfams in terms of AUPR in the MF ontology. Moreover, TANA-1 achieved 18\% improvements over the DiamondBLAST baseline in terms of AUPR in CC ontology. The TANA-1 version obtained better performance on both recall and precision, which indicates the influence of associating network neighborhood information with the shared annotation over BP, CC and MF ontologies. Therefore, the accurate protein-centric or term-centric evaluation of BP depend on data that is not sequence-based or network neighborhood-based, and need expansive strategies like using the Interprodomain, 2D/3D structures and phylogenetic tree for each target protein. For that reason, the orengo-funfams, INGA-Tosatto and DeepGOPLUS got the best results in terms of BP and $\mathrm{CC}$ terms.

Nevertheless, the reason behind the fair performances of the TANA version in predicting CC terms category stands on the fact that the different versions of TANA cannot predict the interlog (i.e., homologous pairs of protein interactions across different organisms) between the proteins for a given specie. Therefore, our approach is not able to predict a conserved interaction across a pair of proteins which have interacting homologs in another species.

Indeed, TANA doesn't leverage the network neighborhood during the process of the prediction, because the alignment of the PPI networks from a myriad of species leads to more noisy prediction coming from different types of intra-cellular location and it got worst if the PPI networks for a given specie is not complete. Therefore, predicting these specific terms yields to a high number of false positives and thus hampers to get a good performance in terms of $F_{\max }$ metric.

\section{Term-centric evaluation}

We can come to the conclusion that, from Table 4, using the different versions of TANA for predicting MF, BP and CC terms (i.e., TANA-1, TANA-3) based only on the transfer of annotation by considering the shared GOA yields us a good performance in terms of termcentric metric than do the TANA-2 and TANA-4. Moreover, to have a good evaluation at the term-centric side, the TANA-1 would be the appropriate version since it discerns well and poorly predictable GO terms. The quality of the prediction for each individual GO terms was evaluated by computing the area under the AUC method. Computing the AUCs for an individual GO term associated to the MF gives us a different insight on the performance evaluation of the GO term belonging to the binding and those with transporter and catalytic 
activity. In general, the association of GO terms with displayed binding function decreases the AUC values.

A precision-recall curve is a plot of the precision (y-axis) and the recall (x-axis) for different thresholds, much like the ROC curve. To assess the accuracy of the prediction model, we plot the ROC curve for each approach. ROC curve represents the worst case with a strong effect of the hazard (AUC of 0.5 associated with a very weak discrimination capacity) and the best case with an AUC of 1 associated with a very strong discrimination capacity. In a ROC curve, true positive rates (y-axis) are plotted and false positive rates (x-axis). The AUC is the area covered by the curve surrounded by a given line generated by the competing methods and the axis. The bigger the area covered, the most suitable the approaches are at differentiating the given classes. As Figure 1 depicts, DeepGO and Naive approach achieved the best score in BP, MF and CC ontologies. The scores are promizing for the different versions of TANA. TANA-1 is roughly above the baseline DiamondBLAST for predicting MF, BP and CC terms. Adding to that, the AUC of both INGA-Tosatto and orengo-funfams outperforms the AUC of the approach DeepGOPLUS for predicting MF, BP and CC terms.

As shown in the Additional file 2: Table S2 and the Additional file 3: Table S3, most of the $F_{\max }$ of GO terms in MF or BP obtained by TANA-1 are better than TANA-3. Whereas, the performance of both approaches are the same by predicting the GO terms in CC (cf. Additional file 4: Table S4). Moreover, among the BP terms, we uncover a null $F_{\max }$ value related to less specific terms such as "cellular process", "response to drug " and "response to insulin". Therefore, the annotation of the function biological macromolecules is a critical task caused by the dominance of poorly predictable GO terms. We also unveil that annotation of the proteins with GO terms in MF, e.g., "antigen binding", "polysaccharide binding", "drug binding" and "macromolecular complex binding" provided high performance. We can also come to the conclusion that the prediction of the MF and CC terms is better performed than the BP category. In addition, we notice that more specific function (GO terms from BP, MF or CC) better perform than the high-level ones. The reason behind this performance can be explained by the influence of the accurate transfer annotation on the quality of the prediction from the GNA and from the direct interaction neighborhood.

Our proposed approach is based on the idea to replace sequence similarities between proteins across different species with their functional similarities, in order to improve the prediction accuracy. Nevertheless, we did not obtain an optimal prediction quality accross the bacterial species, since theirs proteins are not well annotated (cf. Table 1). It is worth mentioning that some coverages (i.e number of target proteins) of TANA, for some species, are low because our proposed approach is basically based on a homology transfer of function prediction. Therefore, this will influence negatively on the quality of the protein-centric or term centric evaluation applied on the NK and LK, and on the "partial-mode" which is based on averaging over the predicted subset. The proteins having no annotation for the $\mathrm{BP}, \mathrm{MF}$ and $\mathrm{CC}$ ontologies belong to the NK category. Whereas, proteins with LK are those that had been experimentally annotated in one or three GO (BP, MF or CC ontology). Meanwhile, the goal is to try to increase the discovery of the number of UA, NMF, NBP, NCC without increasing the value of ME and MNE for each multiple or pairwise alignment (cf. Table 3). 


\section{Limitations of the TANA method and sketch of future works}

The low value of the ROC area is influenced by two factors. Firstly, there are many proteins which are not annotated. Secondly, the worst case when the protein in its PPI network is not annotated and their neighborhood interactions are not complete and have many texts mined and predicted interactions leading to a great number of false positives. In addition, the reason behind the low quality of the BP and CC GO terms consisting in trying to align different species which have distinct PPI networks from different living cells (i.e. species) and have different physical interactors and expected to participate in different biological process and to operate in different subcellular compartment. Therefore, the solution to enhance the quality of the $\mathrm{BP}$ and $\mathrm{CC}$ GO terms, during the prediction process, is to only involve the proteins from the same PPI network and from the same pathway. Another limitation of our approach is the coverage, as it is difficult to produce more predictions whenever the inner neighborhoods of of the target protein are unannotated. On the other side, to boost the coverage and at the same time the prediction accuracy, TANA can integrates a complementary methods (i.e. BLAST) by a consensus approach.

The TANA approach is based on the notion that similar functions are usually caused by similar molecular origins, which is beneficial to reveal the common attributes for each unannotated protein. However, TANA does not profit from the network neighborhood, because focusing only on the direct neighborhood of each target protein is not sufficient. We claim that computing the functional similarity between two compared proteins belonging to a given cluster (from the STRING database) or a given protein complexes (from a manually annotated protein complexes, e.g., the CORUM database) using the GO, DO, HPO would lead automatically to a better prediction accuracy.

Adding to that, focusing on the protein complexes of a target protein causing some given diseases by jointly utilizing gene, disease and phenotype associations, could help researchers gain a deep insight of human diseases, since similar diseases are usually caused by similar phenotypes.

\section{Running time}

All the experiments were carried out on a $2.6 \mathrm{GHz}$ Intel(R) Xeon(R) 48 CPUs, machine running GNU/Linux with 503GB system memory. For the sake of brevity, in this subsection, we only report the running time for the TANA-1 and TANA-2 approaches. So, the required time of the predictions tasks performed by TANA-1 for the datasets "D1" and "D2" was roughly 4 hours and 12 minutes, respectively. Whereas, the time required of TANA-2 for the datasets "D1" and "D2" was roughly 6 hours and 12 minutes, respectively (cf. Table 3).

The reason behind this required time for the dataset "D1" is the given huge number of sequences for each compared species (about roughly 100.000 proteins used during the alignment process), released by the CAFA3 project. Moreover, there are many proteins that have a degree in their PPI network greater than 2000 interactions (e.g. Q5RKN4, Q00420), which should be integrated during the prediction process from the direct interaction neighborhood for a given unannotated protein.

\section{Conclusions}

In this paper, we introduced a new approach for protein-protein function prediction by transferring annotation via PPI networks alignment and from the direct interaction neighbours of the target protein. The approach considers that annotation from BP, MF or CC 
ontologies shared by annotated protein, can be predicted from its interacting partners belonging at the same consistent cluster. The results of the alignment and the prediction of the functionalities of proteins from different species using both GOA and sequence homology are very encouraging and flexible in terms of computational runtime.

Upcoming work, include the integration of the metabolic pathway for each specie during the prediction process. We expect that doing so would give us insight on the different types of reactions involved by each compared protein. Moreover, in terms of quality scores, there is still significant improvement in all ontologies, and particularly in BP and CC terms using different strategies. We also plan to assess the ability of our approach to associate proteins with disease terms from disease gene prediction tasks using the HPO benchmark from CAFA challenge. Finally, we will pay heed to increasing the coverage of the prediction for each species in order to evaluate our approach on the partial mode.

\section{Methods}

We start this section by providing a thorough description of the TANA algorithm.

Shared function associated with the cluster generated by GNA

In the following, we describe our approach for specifying function to an unannotated protein based on the frequency of each GO term within a GNA-aligned cluster of proteins. A functional cluster illustrates a sub-network of proteins that shares a common function. The driving idea of our approach stands on the fact that the functionalities shown by more proteins member, within their cluster, is eligible to prediction.

The algorithm implemented in our approach operates in six major steps (cf., Figure 3):

- Step 1: The function prediction of the annotated protein can be inferred by finding enriched annotations within the cluster by taking into account that each protein from the cluster may be engaged in multiple roles and functions. We extract the GOA induced by a node and its member in the same cluster resulting from GNA.

- Step 2: Following, each annotated protein, within its cluster, is considered suitable for a possible annotation transfer. Among the annotated proteins in the cluster, we consider the proteins that contain at least two GOA overlaps. When modules or clusters are explored, every shared function associated with the module, is used for transferring annotations to the unannotated protein. For this reason, the applied clustering method is mandatory for enhancing the quality of the functional predictions. Interestingly enough, instead of predicting functions for individual proteins, our approach tries, at first, to discover consistent clusters of proteins and then assign functions to all the unannotated proteins in each cluster.

- Step 3: Afterward, TANA finds the shared GOA based on the majority functions of the neighbors in the PPI of an annotated protein and then assigns it these functions.

- Step 4: Next, each function shared by the majority of the clusters' proteins is assigned to all the proteins in the module or in the cluster by putting them into the set of GO terms.

- Step 5 : Then, if the annotation of proteins is not shared by the cluster, then we try to compute the semantic similarity in the context of GO [48] using the Relevance [53] metric. Furthermore, we tried to apply the Jiang and Resnik similarities. However, we obtained a very low coverage which not induce us to assess the performance of the approach because there are a large number of target protein, that have been 
omitted during the prediction. Therefore, in this paper, we only paying heed to the Relevance metric.

- Step 6: After that, we select the compared annotations having the highest confidence score. Therefore, if the given "Relevance metric" between both of compared GO terms is greater than the score 0.5 , then we add them to the list of the predicted terms for each unannotated protein. We set the threshold value of the relevance metric to 0.5 , since it yields the best prediction quality in terms of coverage, protein-centric and term-cenrtic ratio.

To avoid a circular argument in our methodology, we use proteins functional knowledge form the inter-network links (by linking proteins that are functionally similar) that are different from the prediction task:

i) during the alignment process, we exclude only the GOA with evidence code IEA; ii) Secondly, during the prediction task, we only take into consideration the GOA with evidence code EXP, IDA, IEP, IPI, IMP, IGI, TAS and IC.

TANA generates a global alignment from each discovered cluster VertexCluster belonging to the set $V^{*}=\bigcup\{$ VertexCluster $(v)\}: \forall v \in V$. We denote by VertexClusterTOPredict $(u p)$

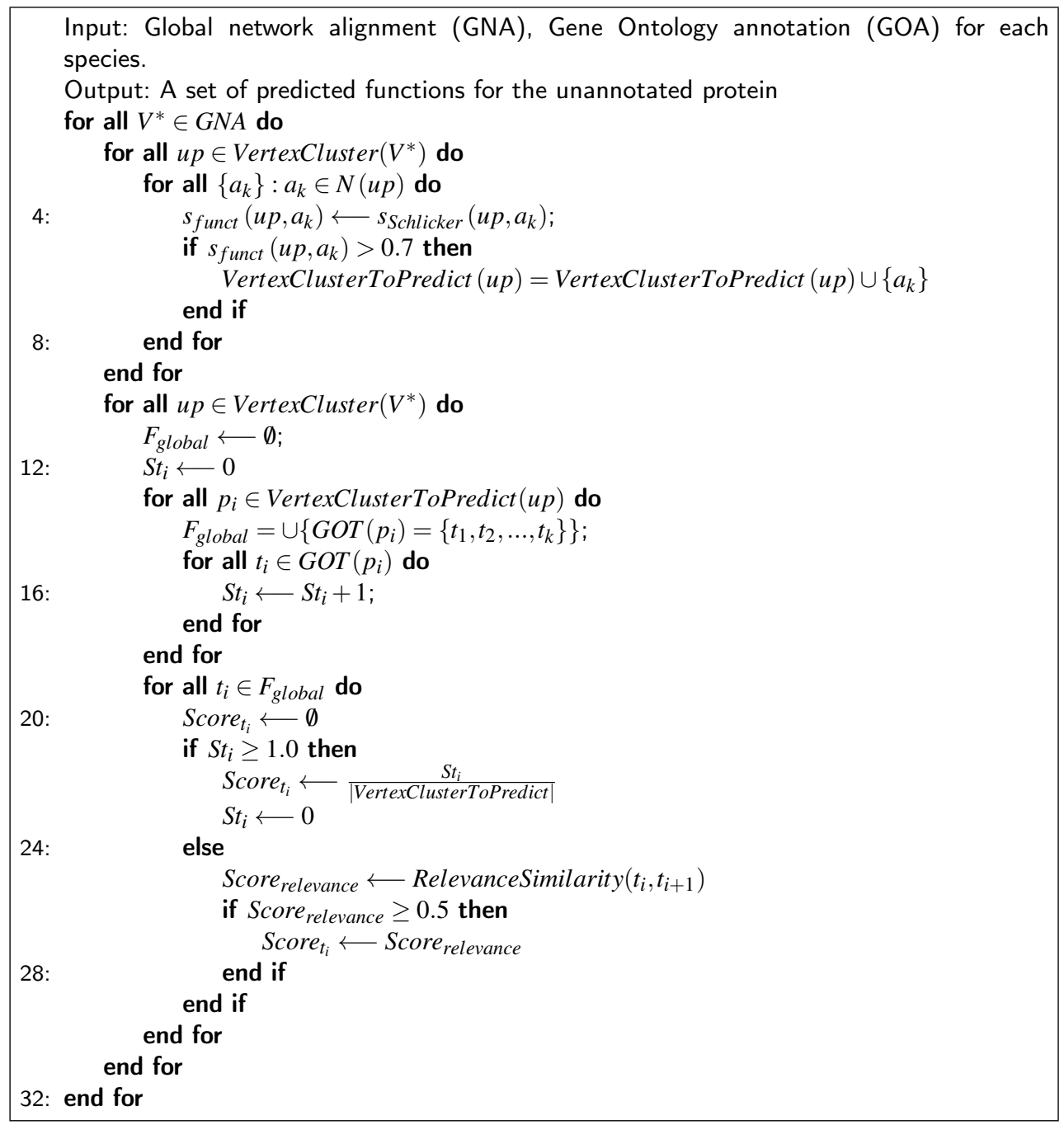

Algorithm 1: FunctionPrediction (GNA,GOA) 
the set of all annotated proteins aligned to the unannotated protein $u p \in V$. Here, GOT $\left(p_{i}\right)$ stands for the set of GO terms annotating a protein $p_{i} \in$ VertexClusterToPredict (up), i.e.,

$$
F_{\text {global }}=\cup\left\{G O T \left(p_{i}=\left\{t_{1}, t_{2}, \ldots, t_{k}\right\}:\right.\right.
$$

$$
\forall p_{i} \in \text { VertexClusterToPredict(up) }
$$

For each function $t_{i} \in G O T\left(p_{i}\right)$ of a given annotated protein $p_{i}$, we assign a score based on the frequency of its occurrence in the $F_{\text {global }}$ list, in order to underscore the collection of the shared functionalities (i.e., GO annotation term) shown by the entire annotated protein member in the cluster or module.

$$
\begin{aligned}
& \text { Score }_{t_{i}}(\text { up })=\frac{\sum_{p_{i} \in \text { VertexClusterToPredict }(\text { up })} \boldsymbol{\delta}\left(p_{i}, t_{i}\right)}{\mid \text { VertexClusterToPredict }(\text { up }) \mid} \\
& \left.\quad \forall t_{i} \in F_{\text {global }} \text { and } \quad \forall p_{i} \in \text { VertexClusterToPredict (up }\right)
\end{aligned}
$$

- 1: $\left(p_{i}, f c t\right)$, if the annotated protein $p_{i}$ has the function $f c t \in G O T\left(p_{i}\right)$;

- 0 : otherwise.

Where $\mid$ VertexClusterToPredict (up)| stands for the number of annotated protein for each predicted cluster.

Afterwards, if the score based on the frequency for each predicted GO term $t_{i}$ is lower than the value 1.0 (i.e., $\operatorname{Score}_{t_{i}}(u p)<1.0$ ), then we try to compute a given semantic similarity (i.e., Resnik, Lin, Jiang, Relevance metric) between the GO term $t_{i}$ versus the other terms $t_{i} \in F_{\text {global }}$. Therefore, if the given "Relevance metric" between both of compared GO terms is greater than the score 0.5 , then we add them to the list of the predicted terms for each unannotated protein $u p$. We set the value 0.5 as the threshold, since it yields us a confidence to ascertain the degree of similarity between the compared GO terms. We applied this method in order to stress on the importance of GO terms that give a good score using the semantic similarities, even if the score of the two compared terms is low in terms of frequency (i.e., Score $_{t_{i}}($ up $)$ ) (cf., Algorithm 1).

We perform the prediction task by assuming that the GNA results is with a higher evaluation in terms of total coverage and consistency between clusters [45]. Therefore, to assess the biologically relevance of the clusters, we consider the clusters having at least two annotated proteins (using the biological process or molecular function annotations) as well as the cluster that yields good scores on two key dimensions: coverage and consistency. Then, the evaluation is of paramount importance in order to ascertain the biologically relevance to be used to transfer annotations, after the evaluation of the all PPI network alignment paradigms. For the sake of brevity, we took an example of some proteins, in order to explain more our strategy. Meanwhile, our approach can process another cases with more proteins than six or seven proteins. Thus, Figure 4 depicts the three cases encountered by our approach to predict the function of the unannotated protein;

1 Case 1: When TANA encounters a cluster with 6 proteins, only two of them are with one known shared function (i.e., yellow ovals). Then, our approach blindly assigns the shared function to the four unannotated proteins (i.e., white ovals);

2 Case 2: When TANA encounters a cluster with 6 proteins, five of them are annotated with two known shared functions (i.e., yellow and blue ovals). Then, our approach blindly assigns both of the shared functions to the unannotated protein; 
3 Case 3: When TANA encounters a cluster with seven proteins, six of them are annotated with two known shared functions (i.e., yellow and blue ovals) and two different functions (i.e., green and red ovals) but they are related semantically with the other shared functions by applying the different functional similarities (i.e., Resnik, Lin, Jiang or Relevance similarity). Then, our approach blindly assigns the two shared functions to the unannotated protein and semantically the two other functions (i.e., green and red ovals).

The shared function associated with network neighbors of the unannotated protein The degree of a protein is the amount of interactions in the PPI network. If a given protein has a larger degree then it means that form two types of functional module: a) protein complexes (simultaneous interactions); b) metabolic pathways (consecutive interactions). The proteins do not accomplish their biological functions by themselves but achieve them by interrelating with other proteins or with copies of themselves in PPIs networks. The idea of shared network neighbors has been used: proteins that share many common neighbors also have close functional associations (cf., Figure 5). For each given protein, we computed how many times each of the shared functions occur as annotation in the direct interaction neighbourhood of the protein.

For a given unannotated protein $u p \in V$, we denote the PPI neighborhood of $u p, N(u p)$, to be the subgraph of $G$ whose vertex set consists of all of interaction neighbors passed over up. Formally, we set $N(u p)=\left(V_{u p}, E_{u p}, W_{u p}\right)$, where $V_{u p}=\{v \in V \mid(u p, v) \in E\}$, $E_{u p}=\left\{\left(v, v^{\prime}\right) \in E \mid(u p, v) \in E\right.$ and $\left.\left(u p, v^{\prime}\right) \in E\right\}$, and $W_{u p}$ are the weight on the edges in $E_{u p}$. Then, we try to compute the functional similarity using the SCHLICKER metric in order to select the compared protein having the highest confidence score (greater than 0.7 ), then we add the annotated protein from the direct network neighborhood to the list VertexClusterToPredict (up) of the cluster for each up unannotated protein. Finally, the added annotated protein from the neighbour of $u p$ will share its GO terms within the cluster or the module which up belong (cf. Algorithm 1). This technique will enhance the results of the CC GO terms, because this process is based on the discovering of the direct interaction neighbours inner the same specie of $u p$.

\section{Theoretical Complexity Study}

The complexity of TANA depends on the number of $k$ network, which should be aligned. Adding to that, TANA applies the strategy of the shared GO terms with the comparison of the network neighbours for each unknown proteins using the SCHLICKER functional similarity. So, for each $k$ networks, where the maximum network size is $n=\max _{i}\left|V_{i}\right|$, the maximum number of interactions in a network is $m=\max _{i}\left|E_{i}\right|$. For each bipartite graph, $B_{s}=\left(V_{s 1} \cup V_{s 2}, E_{s}\right)$ the running time complexity on $B_{s}$ is about $O\left(\left|V_{s 1} \cup V_{s 2}\right| \cdot \log \left|E_{s}\right|\right)$. Adding to that, the collection of candidate edge $\left(\begin{array}{l}k \\ 2\end{array}\right) O(n \log (n))$ time. Finally, after the running of the Simulated Annealing [45], the function prediction task depends on the density of the network neighbour which is about $O(n)$.

\footnotetext{
Abbreviations

TANA: Transferring Annotation via Network Alignment; PPI: Protein-Protein Interaction; CAFA: Computational Assessment of Function Annotation; SVMs: Support Vector Machines; MSA: Multiple sequence alignment; FDRs: Functionally discriminating residues; PSSM: Position specific scoring matrix; LTR: Learning to rank; MF: Molecular function; BP: Biological process; CC:Cellular component; GNA: Global network
} 
Alignment; GOA: Gene ontology annotation; GO: Gene ontology; STRING: Search Tool for the Retrieval of Interacting Genes/Proteins; UniProtKB:UniProt Knowledgebase; ROC:Receiver Operating Characteristic; AUC Area Under Curve; AUPR: Area under the precision-recall curve; NK: No-knowledge; LK: Limited-knowledge; HPO: Human Phenotype Ontology; DO: Disease Ontology; IEA: Inferred from Electronic Annotation; EXP: Inferred from Experiment; IDA: Inferred from Direct Assay; IEP: Inferred from Expression Pattern; IPI: Inferred from Physical Interaction; IMP: Inferred from Mutant Phenotype; IGI: Inferred from Genetic Interaction; TAS:Traceable Author Statement; IC: Inferred by Curator.

Declarations

Ethics approval and consent to participate

Not applicable.

Consent for publication

Not applicable.

Availability and requirements

Project name: TANA

Project home page: http://doi.org/10.5281/zenodo.3718451

Operating system(s): Linux

Programming language: $\mathrm{C}++$

Other requirements: GCC compiler

License: BSD 3-Clause

Any restrictions to use by non-academics: N/A

Availability of data and material

The $\mathrm{C}++$ source code written for this publication and the datasets used to generate the results is accessible through http://doi.org/10.5281/zenodo.3718451. The python code to evaluate our approach and its competitors is available at http://doi.org/10.5281/zenodo.3726187 under the BSD 3-Clause License.

Competing interests

The authors declare that they have no competing interests.

Funding

Not applicable.

Authors' contributions

EMN and SBY initiated the study. WDJ developed the code, conducted the experiments and wrote the manuscript. All authors read and approved the final version.

Acknowledgments

Not applicable.

Author details

${ }^{1}$ University of Tunis EI Manar, Faculty of Sciences of Tunis, LR11ES14, Capmus Universitaire, 2092 Tunis Tunisia. ${ }^{2}$ Tallinn University of Technology, Department of Software Science, Akadeemia tee 15a, 12618,

Tallinn, Estonia. ${ }^{3}$ University Clermont Auvergne, CNRS, LIMOS, F-63000 CLERMONT-FERRAND, FRANCE.

References

1. Tran, L.: Hypergraph and protein function prediction with gene expression data. arXiv preprint arXiv:1212.0388 (2012)

2. Kotaru, A.R., Joshi, R.C.: Classification of phylogenetic profiles for protein function prediction: An svm approach. In: International Conference on Contemporary Computing, pp. 510-520 (2009). Springer

3. Morin, M.M.: Phylogenetic Networks: Simulation, Characterization, and Reconstruction, (2007)

4. Sael, L., Chitale, M., Kihara, D.: Structure-and sequence-based function prediction for non-homologous proteins. Journal of structural and functional genomics 13(2), 111-123 (2012)

5. Wang, Z., Cao, R., Cheng, J.: Three-level prediction of protein function by combining profile-sequence search, profile-profile search, and domain co-occurrence networks. In: BMC Bioinformatics, vol. 14, p. 3 (2013). BioMed Central

6. Laskowski, R.A., Watson, J.D., Thornton, J.M.: Protein function prediction using local 3d templates. Journal of molecular biology 351(3), 614-626 (2005)

7. Glazer, D.S., Radmer, R.J., Altman, R.B.: Improving structure-based function prediction using molecular dynamics. Structure 17(7), 919-929 (2009)

8. Zhao, X.-M., Chen, L., Aihara, K.: Protein function prediction with the shortest path in functional linkage graph and boosting. International journal of bioinformatics research and applications 4(4), 375-384 (2008)

9. Sharan, R., Ulitsky, I., Shamir, R.: Network-based prediction of protein function. Molecular systems biology 3(1), 88 (2007)

10. Zhou, X., Kao, M.-C.J., Wong, W.H.: Transitive functional annotation by shortest-path analysis of gene expression data. Proceedings of the National Academy of Sciences 99(20), 12783-12788 (2002) 
11. Wu, J., Kasif, S., DeLisi, C.: Identification of functional links between genes using phylogenetic profiles. Bioinformatics 19(12), 1524-1530 (2003)

12. Altschul, S.F., Gish, W., Miller, W., Myers, E.W., Lipman, D.J.: Basic local alignment search tool. Journal of molecular biology 215(3), 403-410 (1990)

13. Benso, A., Di Carlo, S., ur Rehman, H., Politano, G., Savino, A., Suravajhala, P.: A combined approach for genome wide protein function annotation/prediction. Proteome science 11(1), 1 (2013)

14. Wang, K., Samudrala, R.: Fssa: a novel method for identifying functional signatures from structural alignments. Bioinformatics 21(13), 2969-2977 (2005)

15. Ferré, S., King, R.D.: Finding motifs in protein secondary structure for use in function prediction. Journal of Computational Biology 13(3), 719-731 (2006)

16. Erdin, S., Venner, E., Lisewski, A.M., Lichtarge, O.: Function prediction from networks of local evolutionary similarity in protein structure. In: BMC Bioinformatics, vol. 14, p. 6 (2013). BioMed Central

17. Laskowski, R.A., Watson, J.D., Thornton, J.M.: Profunc: a server for predicting protein function from 3d structure. Nucleic acids research 33(suppl_2), 89-93 (2005)

18. Deng, M., Zhang, K., Mehta, S., Chen, T., Sun, F.: Prediction of protein function using protein-protein interaction data. Journal of computational biology 10(6), 947-960 (2003)

19. Letovsky, S., Kasif, S.: Predicting protein function from protein/protein interaction data: a probabilistic approach. Bioinformatics 19(suppl 1), 197-204 (2003)

20. Jaeger, S., Gaudan, S., Leser, U., Rebholz-Schuhmann, D.: Integrating protein-protein interactions and text mining for protein function prediction. In: BMC Bioinformatics, vol. 9, p. 2 (2008). BioMed Central

21. Ahmed, K.S., Saloma, N.H., Kadah, Y.M.: Improving the prediction of yeast protein function using weighted protein-protein interactions. Theoretical Biology and Medical Modelling 8(1), 11 (2011)

22. Xiong, W., Liu, H., Guan, J., Zhou, S.: Protein function prediction by collective classification with explicit and implicit edges in protein-protein interaction networks. BMC bioinformatics 14(12), 4 (2013)

23. Peng, W., Wang, J., Cai, J., Chen, L., Li, M., Wu, F.-X.: Improving protein function prediction using domain and protein complexes in ppi networks. BMC systems biology 8(1), 35 (2014)

24. Wu, Q., Ye, Y., Ng, M.K., Ho, S.-S., Shi, R.: Collective prediction of protein functions from protein-protein interaction networks. In: BMC Bioinformatics, vol. 15, p. 9 (2014). BioMed Central

25. Yu, G., Zhu, H., Domeniconi, C., Guo, M.: Integrating multiple networks for protein function prediction. In: BMC Systems Biology, vol. 9, p. 3 (2015). BioMed Central

26. Chua, H.N., Sung, W.-K., Wong, L.: An efficient strategy for extensive integration of diverse biological data for protein function prediction. Bioinformatics 23(24), 3364-3373 (2007)

27. Kuchaiev, O., Pržulj, N.: Integrative network alignment reveals large regions of global network similarity in yeast and human. Bioinformatics 27(10), 1390-1396 (2011)

28. Singh, R., Xu, J., Berger, B.: Global alignment of multiple protein interaction networks with application to functional orthology detection. Proceedings of the National Academy of Sciences (2008)

29. Sharan, R., Ideker, T.: Modeling cellular machinery through biological network comparison. Nature biotechnology 24(4), 427 (2006)

30. Cozzetto, D., Minneci, F., Currant, H., Jones, D.T.: Ffpred 3: feature-based function prediction for all gene ontology domains. Scientific reports 6, 31865 (2016)

31. Gong, Q., Ning, W., Tian, W.: Gofdr: a sequence alignment based method for predicting protein functions. Methods 93, 3-14 (2016)

32. You, R., Huang, X., Zhu, S.: Deeptext2go: Improving large-scale protein function prediction with deep semantic text representation. Methods 145, 82-90 (2018)

33. You, R., Zhang, Z., Xiong, Y., Sun, F., Mamitsuka, H., Zhu, S.: Golabeler: improving sequence-based large-scale protein function prediction by learning to rank. Bioinformatics 34(14), 2465-2473 (2018)

34. You, R., Yao, S., Xiong, Y., Huang, X., Sun, F., Mamitsuka, H., Zhu, S.: Netgo: improving large-scale protein function prediction with massive network information. Nucleic acids research 47 (W1), 379-387 (2019)

35. Kulmanov, M., Khan, M.A., Hoehndorf, R.: Deepgo: predicting protein functions from sequence and interactions using a deep ontology-aware classifier. Bioinformatics 34(4), 660-668 (2017)

36. Piovesan, D., Giollo, M., Leonardi, E., Ferrari, C., Tosatto, S.C.E.: Inga: protein function prediction combining interaction networks, domain assignments and sequence similarity. Nucleic Acids Research 43(W1), 134-140 (2015). doi:10.1093/nar/gkv523

37. Finn, R.D., Bateman, A., Clements, J., Coggill, P., Eberhardt, R.Y., Eddy, S.R., Heger, A., Hetherington, K., Holm, L., Mistry, J., et al.: Pfam: the protein families database. Nucleic acids research 42(D1) 222-230 (2013)

38. Franceschini, A., Szklarczyk, D., Frankild, S., Kuhn, M., Simonovic, M., Roth, A., Lin, J., Minguez, P., Bork, P., Von Mering, C., et al.: String v9. 1: protein-protein interaction networks, with increased coverage and integration. Nucleic acids research 41(D1), 808-815 (2012)

39. Piovesan, D., Tosatto, S.C.E.: INGA 2.0: improving protein function prediction for the dark proteome. Nucleic Acids Research 47(W1), 373-378 (2019). doi:10.1093/nar/gkz375. https://academic.oup.com/nar/article-pdf/47/W1/W373/28879989/gkz375.pdf

40. Kulmanov, M., Hoehndorf, R.: DeepGOPlus: improved protein function prediction from sequence. Bioinformatics 36(2), 422-429 (2019). doi:10.1093/bioinformatics/btz595. https://academic.oup.com/bioinformatics/article-pdf/36/2/422/31962785/btz595.pdf

41. Scheibenreif, L., Littmann, M., Orengo, C., Rost, B.: Funfam protein families improve residue level molecular function prediction. BMC bioinformatics 20(1), 1-9 (2019)

42. Fellenberg, M., Albermann, K., Zollner, A., Mewes, H.-W., Hani, J., et al.: Integrative analysis of protein interaction data. In: Ismb, vol. 8, pp. 152-161 (2000)

43. Hishigaki, H., Nakai, K., Ono, T., Tanigami, A., Takagi, T.: Assessment of prediction accuracy of protein function from protein-protein interaction data. Yeast 18(6), 523-531 (2001) 
44. Hawkins, T., Kihara, D.: Function prediction of uncharacterized proteins. Journal of bioinformatics and computational biology 5(01), 1-30 (2007)

45. W. E., D., Ben-Yahia, S., Mephu-Nguifo, E.: A novel computational approach for global alignment for multiple biological networks. IEEE/ACM Transactions on Computational Biology and Bioinformatics 15(6), 2060-2066 (2018)

46. Schlicker, A., et al.: A new measure for functional similarity of gene products based on gene ontology. BMC Bioinformatics 7(1), 302 (2006). doi:10.1186/1471-2105-7-302

47. Szklarczyk, D., Franceschini, A., Wyder, S., Forslund, K., Heller, D., Huerta-Cepas, J., Simonovic, M. Roth, A., Santos, A., Tsafou, K.P., et al.: String v10: protein-protein interaction networks, integrated over the tree of life. Nucleic acids research 43(D1), 447-452 (2014)

48. Pesquita, C., Faria, D., Bastos, H., Ferreira, A.E., Falcão, A.O., Couto, F.M.: Metrics for go based protein semantic similarity: a systematic evaluation. In: BMC Bioinformatics, vol. 9, p. 4 (2008). BioMed Central

49. Zhou, N., Jiang, Y., Bergquist, T.R., Lee, A.J., Kacsoh, B.Z., Crocker, A.W., Lewis, K.A., Georghiou, G., Nguyen, H.N., Hamid, M.N., et al.: The cafa challenge reports improved protein function prediction and new functional annotations for hundreds of genes through experimental screens. Genome biology 20(1), 1-23 (2019)

50. Clark, W.T., Radivojac, P.: Information-theoretic evaluation of predicted ontological annotations. Bioinformatics 29(13), 53-61 (2013)

51. Davis, J., Goadrich, M.: The relationship between precision-recall and roc curves. In: Proceedings of the 23rd International Conference on Machine Learning, pp. 233-240 (2006). ACM

52. Kulmanov, M., Hoehndorf, R.: Deepgoplus: Improved protein function prediction from sequence. bioRxiv, 615260 (2019)

53. Schlicker, A., Domingues, F.S., Rahnenführer, J., Lengauer, T.: A new measure for functional similarity of gene products based on gene ontology. BMC bioinformatics 7(1), 302 (2006)

Additional Files

Additional file 1 Table S1 - The different pairwise alignment strategies on the Eukaryotes and Prokayotes species used by TANA

Additional file 2 Table S2 - Evaluation of TANA for each GO term (Biological Process), on the different organisms of CAFA3 evaluation set (full evaluation)

Additional file 3 Table S3 - Evaluation of TANA for each GO term (Molecular Function), on the different organisms of CAFA3 evaluation set (full evaluation)

Additional file 4 Table S4 - Evaluation of TANA for each GO term (Cellular Component), on the different organisms of CAFA3 evaluation set (full evaluation)

Figure Legends

[width $=120 \mathrm{~mm}$ ]Figure1-a.eps $[$ width $=120 \mathrm{~mm}]$ Figure1-b.eps $[$ width $=120 \mathrm{~mm}]$ Figure1-c.eps

(a)

(b) (c)

Figure 1: The ROC curves of TANA and other predictors over BPO, MFO and CCO in full evaluation mode.

[width $=120 \mathrm{~mm}$ ]Figure2-a.eps [width $=120 \mathrm{~mm}$ ]Figure2-b.eps [width $=120 \mathrm{~mm}$ ]Figure2-c.eps

(a)

(b)

(c)

Figure 2: The precision-recall plot of TANA and other predictors over BPO, MFO and $\mathrm{CCO}$ in full evaluation mode.

[width $=7 \mathrm{~cm}$, height $=7 \mathrm{~cm}]$ Figure3.eps

Figure 3: The different steps of TANA for scoring the prediction of GO terms for each unannotated protein.

[width $=9 \mathrm{~cm}$, height $=7 \mathrm{~cm}]$ Figure4.eps

Figure 4: Functional annotation yield by TANA. This shows clusters of proteins composed from unannotated proteins as well as annotated ones 
[width $=15 \mathrm{~cm}$, height $=12 \mathrm{~cm}]$ Figure5.eps

Figure 5: Two different strategies of protein function prediction are yield by TANA. In the PPI network on the left, proteins A, E, and F are annotated with "green " function, proteins $C$ and $D$ are annotated with "blue" function. The node $H$ is annotated with the "yellow" function and G and B are unannotated proteins (as denoted by white node color). With the "direct neighborhood" approach (the middle panel), node $B$ gets annotated with "green" function, since the majority of its neighbors ( $E$ and F) have the "green" function. Similarly, G gets annotated with "blue" function, since all of its neighbors ( $C$ and $D$ ) have the "blue" function. Adding to that, by using the other strategy based on transferring annotation from the alignment of PPI network, the protein B is also annotated with two shared functions (i.e, orange and black colors) coming from the "Cluster 1 ". Moreover, protein G also gets annotated with one shared function (i.e., Black color) from the shared function of the "Cluster 2 ". It is worth mentioning that: a) the proteins with a shape different from the circle node are a proteins coming from different species; b) The proteins $B$ and $G$ are the proteins with limited Knowledge, which had been experimentally annotated in one or two GO ontologies (BP, MF or CC).

Tables

Table 1: Characteristics of the PPI Networks from 18 Species.

\begin{tabular}{|c|c|c|c|c|c|c|c|}
\hline \multicolumn{2}{|c|}{ Domain Taxonomyame } & \multirow{2}{*}{$\begin{array}{l}\text { Number } \\
\text { of pro- } \\
\text { teins } \\
12792\end{array}$} & \multirow{2}{*}{$\begin{array}{l}\text { Number } \\
\text { of inter- } \\
\text { actions } \\
1942502\end{array}$} & \multirow{2}{*}{$\begin{array}{l}\text { MF } \\
2776\end{array}$} & \multirow{2}{*}{$\begin{array}{l}\mathrm{BP} \\
5815\end{array}$} & \multirow{2}{*}{$\begin{array}{l}\text { CC } \\
3963\end{array}$} & \multirow{2}{*}{$\begin{array}{l}\text { Largest } \\
\text { de- } \\
\text { gree } \\
2873\end{array}$} \\
\hline Eukaryota7227 & $\begin{array}{l}\text { Drosophila } \\
\text { melanogaster }\end{array}$ & & & & & & \\
\hline Eukaryota239 & $\begin{array}{l}\text { Caenorhabditis ele- } \\
\text { gans }\end{array}$ & 17522 & 3640543 & 1740 & 2575 & 2197 & 3708 \\
\hline Eukaryotæ 906 & Homo sapiens & 19080 & 5867186 & 12202 & 11036 & 12581 & 7613 \\
\hline Eukaryotal10090 & MOUSE & 16049 & 5598454 & 3765 & 4604 & 6886 & 8512 \\
\hline Eukaryotal0116 & RAT & 17139 & 6444811 & 2534 & 3485 & 3612 & 15261 \\
\hline Eukaryota3702 & ARATH & 19080 & 5787443 & 4947 & 6979 & 8380 & 6753 \\
\hline Eukaryota44689 & DICDI & 9737 & 1387080 & 325 & 450 & 777 & 2597 \\
\hline EukaryotaA932 & YEAST & 6492 & 919449 & 3933 & 4632 & 4035 & 2188 \\
\hline Eukaryota7955 & DANRE & 22291 & 6945346 & 670 & 833 & 570 & 6072 \\
\hline Archaea 243232 & $\begin{array}{l}\text { Methanocaldococcus } \\
\text { jannaschii }\end{array}$ & 1751 & 178286 & 46 & 25 & 3 & 697 \\
\hline Archaea 273057 & $\begin{array}{l}\text { Sulfolobus solfataricus } \\
\text { P2 }\end{array}$ & 2858 & 299521 & 22 & 10 & 0 & 1027 \\
\hline Bacteria 160488 & $\begin{array}{l}\text { Pseudomonas putida } \\
\text { KT2440 }\end{array}$ & 5163 & 745882 & 19 & 13 & 0 & 1815 \\
\hline Bacteria 170187 & $\begin{array}{l}\text { Streptococcus } \\
\text { moniae } \\
4\end{array}$ & 2083 & 153530 & 0 & 0 & 0 & 835 \\
\hline Bacteria 223283 & $\begin{array}{l}\text { Pseudomonas syringae } \\
\text { pv. tomato }\end{array}$ & 5318 & 653393 & 15 & 15 & 17 & 1742 \\
\hline Bacteria 224308 & Bacilus subtilis & 4161 & 509967 & 209 & 145 & 32 & 2012 \\
\hline Bacteria 243273 & $\begin{array}{l}\text { Mycoplasma genital- } \\
\text { ium }\end{array}$ & 474 & 30419 & 1 & 2 & 0 & 318 \\
\hline Bacteria 316407 & $\begin{array}{l}\text { Escherichia coli str. K- } \\
12\end{array}$ & 4140 & 386303 & 2264 & 2201 & 2079 & 1453 \\
\hline Bacteria 85962 & Helicobacter pylori & 1461 & 82068 & 89 & 16 & 13 & 624 \\
\hline
\end{tabular}


Table 2: Statistics on the species used by CAFA3

\begin{tabular}{|c|c|c|c|c|c|c|c|c|c|c|}
\hline Domain & Taxonor & NDame & Counts & $\mathrm{BP}(\mathrm{LM})$ & CC (LM) & $\begin{array}{l}\text { MF } \\
\text { (LM) }\end{array}$ & FULL & $\mathrm{NO}$ & D1 & D2 \\
\hline Eukaryota & 7227 & $\begin{array}{l}\text { Drosophila } \\
\text { melanogaster }\end{array}$ & 13907 & - & - & - & - & - & $x$ & \\
\hline Bacteria & 208963 & $\begin{array}{l}\text { Pseudomonas aerugi- } \\
\text { nosa UCBPP PA14 }\end{array}$ & 5892 & - & - & - & - & - & & $*$ \\
\hline Eukaryota & 237561 & $\begin{array}{ll}\text { Candida } & \text { albicans } \\
\text { SC5314 } & \\
\end{array}$ & 12421 & - & - & - & - & - & $*$ & \\
\hline Eukaryota & 9606 & Homo sapiens & 20197 & 4282 & 1905 & 5021 & 4647 & 8266 & $x$ & \\
\hline Eukaryota & 10090 & MOUSE & 16806 & 2296 & 2994 & 5221 & 3005 & 6850 & $x$ & \\
\hline Eukaryota & 10116 & RAT & 7963 & 824 & 1112 & 1354 & 1920 & 3781 & $x$ & \\
\hline Eukaryota & 284812 & SCHPO & 5120 & 2939 & 67 & 3745 & 679 & 582 & * & \\
\hline Eukaryota & 3702 & ARATH & 14754 & 2727 & 2136 & 5084 & 1899 & 6464 & $x$ & \\
\hline Eukaryota & 44689 & DICDI & 4131 & 198 & 315 & 587 & 218 & 3203 & $x$ & \\
\hline Eukaryota & 559292 & YEAST & 6721 & 430 & 983 & 1704 & 2426 & 1801 & $x$ & \\
\hline Eukaryota & 7955 & DANRE & 2967 & 42 & 709 & 672 & 49 & 2113 & $x$ & \\
\hline Eukaryota & 8355 & Xenopus laevis & 3402 & 72 & 230 & 201 & 84 & 2996 & $*$ & \\
\hline Archaea & 243232 & $\begin{array}{l}\text { Methanocaldococcus } \\
\text { jannaschii }\end{array}$ & 1787 & 22 & 45 & 5 & 3 & 1739 & & $x$ \\
\hline Archaea & 273057 & $\begin{array}{l}\text { Sulfolobus solfataricus } \\
\text { P2 }\end{array}$ & 469 & 4 & 14 & 0 & 0 & 455 & & $\times$ \\
\hline Bacteria & 160488 & $\begin{array}{l}\text { Pseudomonas putida } \\
\text { KT2440 }\end{array}$ & 705 & 3 & 16 & 0 & 0 & 689 & & $x$ \\
\hline Bacteria & 170187 & $\begin{array}{lr}\text { Streptococcus } & \text { pneu- } \\
\text { moniae } & \text { serotype } \\
4 & \end{array}$ & 501 & 5 & 4 & 1 & 0 & 496 & & $x$ \\
\hline Bacteria & 223283 & $\begin{array}{l}\text { Pseudomonas syringae } \\
\text { pv. tomato }\end{array}$ & 678 & 0 & 1 & 1 & 0 & 677 & & $x$ \\
\hline Bacteria & 224308 & Bacilus subtilis & 4185 & 51 & 166 & 83 & 8 & 3987 & & $\times$ \\
\hline Bacteria & 243273 & $\begin{array}{l}\text { Mycoplasma genital- } \\
\text { ium }\end{array}$ & 483 & 0 & 2 & 1 & 0 & 481 & & $x$ \\
\hline Bacteria & 321314 & $\begin{array}{l}\text { Salmonella cholerae- } \\
\text { suis }\end{array}$ & 882 & 0 & 0 & 0 & 0 & 882 & & $*$ \\
\hline Bacteria & 83333 & Escherichia coli & 4434 & 978 & 1105 & 1130 & 1056 & 1242 & & $x$ \\
\hline Bacteria & 85962 & Helicobacter pylori & 593 & 7 & 17 & 12 & 0 & 573 & & $x$ \\
\hline Bacteria & 99287 & $\begin{array}{l}\text { Salmonella } \\
\text { phimurium }\end{array}$ & 1789 & 11 & 36 & 22 & 7 & 1733 & & $*$ \\
\hline
\end{tabular}

Note:

FULL : Number of proteins that have experimental annotations in all three ontologies (BP, MF or CC ontology)

NO: Number of proteins that have no experimental annotations in any ontology.

* : The specie mentionned by ' $*$ ' indicates that it does not have a PPI network in the STRING database

- : The number of annotations for the proteins belonging to a given specie is not supplied by the CAFA3

LM : refers to limited

Table 3: Comparative Consistency on the Different PPI Networks.

\begin{tabular}{|c|c|c|c|c|c|c|c|c|}
\hline Datasets & $\mathrm{CV}(\%)$ & $\mathrm{ME}$ & MNE & Time & UA & NMF & NBP & NCC \\
\hline \multicolumn{9}{|c|}{$\begin{array}{c}\text { TANA-1 } \\
\end{array}$} \\
\hline D1 & 38.60 & 0.938 & 0.373 & $\approx 4 \mathrm{~d}$ & 15349 & 7788 & 9420 & 8658 \\
\hline D2 & 7.360 & 0.330 & 0.230 & $5 \mathrm{~min}$ & 373 & 202 & 156 & 176 \\
\hline \multicolumn{9}{|c|}{ TANA-2 } \\
\hline D1 & 38.50 & 0.929 & 0.370 & $\approx 6 \mathrm{~h}$ & 15251 & 7703 & 9479 & 8680 \\
\hline D2 & 7.38 & 0.337 & 0.237 & $3.1 \mathrm{~min}$ & 373 & 202 & 156 & 176 \\
\hline
\end{tabular}

Note: the algorithms TANA-1 and TANA-2 were tested on the two datasets D1 and D2. Each row lists the coverage (CV), mean entropy (ME), meannormalized entropy (MNE), the running time, unknown alignment (UA) and the number of proteins annotated with NMF, NBP and NCC in alignment graph respectively. "Sec", "min", " $h$ " and "d" in the row of time represent seconds, minutes, hours and days. 
Table 4: Evaluation of TANA, DeepGO, DeepGOPLUS, orengo-funfams, INGA-Tosatto, DiamondScore, DiamondBLAST, Naive and GoFDR methods on the CAFA3 evaluation set (NK benchmarks / Full evaluation)

\begin{tabular}{|c|c|c|c|c|c|c|c|c|c|c|c|c|c|}
\hline \multirow{2}{*}{ Methods } & \multicolumn{4}{|l|}{ BP } & \multicolumn{4}{|l|}{ MF } & \multicolumn{5}{|l|}{$\mathrm{CC}$} \\
\hline & $F_{\max }$ & $A U P R$ & AUC & $\mathrm{MCC}$ & $F_{\max }$ & $A U P R$ & AUC & $\mathrm{MCC}$ & $F_{\max }$ & $S_{\min }$ & $A U P R$ & AUC & $\mathrm{MCC}$ \\
\hline DiamondBLAST & 0.399 & 25.3200 .116 & 0.738 & 0.156 & 0.431 & 10.2330 .178 & 0.608 & 0.074 & 0.506 & 8.800 & 0.142 & 0.731 & $\overline{0.146}$ \\
\hline & 0.427 & 22.8600 .267 & 0.828 & 0.314 & .509 & $\begin{array}{ll}9.031 & 0.340\end{array}$ & 0.774 & 0.239 & 0.557 & 8.198 & 0.335 & 0.837 & $\overline{0.260}$ \\
\hline & .357 & 25.0280 .254 & 0.966 & 0.245 & .290 & 10.7330 .130 & 0.963 & 0.132 & 0.562 & 8.465 & 0.456 & 0.979 & 0.321 \\
\hline TA & 0.362 & 33.2940 .219 & 0.776 & 0.228 & .630 & $9.271 \quad 0.491$ & 0.656 & 0.120 & 0.477 & 7.486 & 0.320 & 0.710 & 0.000 \\
\hline TAN & 0.354 & 34.0490 .160 & 0.767 & 0.270 & 0.549 & 12.3310 .371 & 0.723 & 0.317 & 0.445 & 9.321 & 0.277 & 0.694 & 0.280 \\
\hline & 339 & 35.5260 .170 & 0.743 & 0.190 & 0.533 & 10.9230 .356 & 0.690 & 0.277 & 0.487 & 9.376 & 0.317 & 0.675 & 0.189 \\
\hline & 333 & 36.3390 .151 & 0.752 & 0.244 & 0.493 & 12.6180 .321 & 0.731 & 0.098 & 0.470 & 9.358 & 0.265 & 0.702 & 0.272 \\
\hline & 0.195 & 37.4510 .048 & 0.674 & 0.000 & 0.190 & 10.1290 .024 & 0.803 & 0.471 & 0.465 & 11.567 & 70.225 & 0.730 & 0.372 \\
\hline DeepGO & 0.375 & 33.4870 .312 & 0.887 & 0.338 & 0.303 & $9.153 \quad 0.198$ & 0.889 & 0.361 & 0.561 & 9.706 & 0.548 & 0.955 & 0.531 \\
\hline Dee & 0.469 & 22.5730 .404 & 0.828 & 0.297 & 0.544 & 8.724 & 0.774 & 0.222 & 0.623 & 7.823 & 0.627 & 0.837 & 0.260 \\
\hline o-tuntams & 0.531 & 23.2730 .386 & 0.842 & 0.456 & 0.606 & 7.651 & 0.837 & 0.482 & 0.646 & 8.263 & 0.440 & 0.884 & 0.576 \\
\hline INGA-Tosatto & 0.506 & 23.8750 .247 & 0.850 & 0.440 & 0.548 & 8.128 & 0.846 & 0.466 & 0.612 & 8.735 & 0.518 & 0.853 & 0.524 \\
\hline
\end{tabular}

Note: Best results are indicated in bold with respect to each column. 

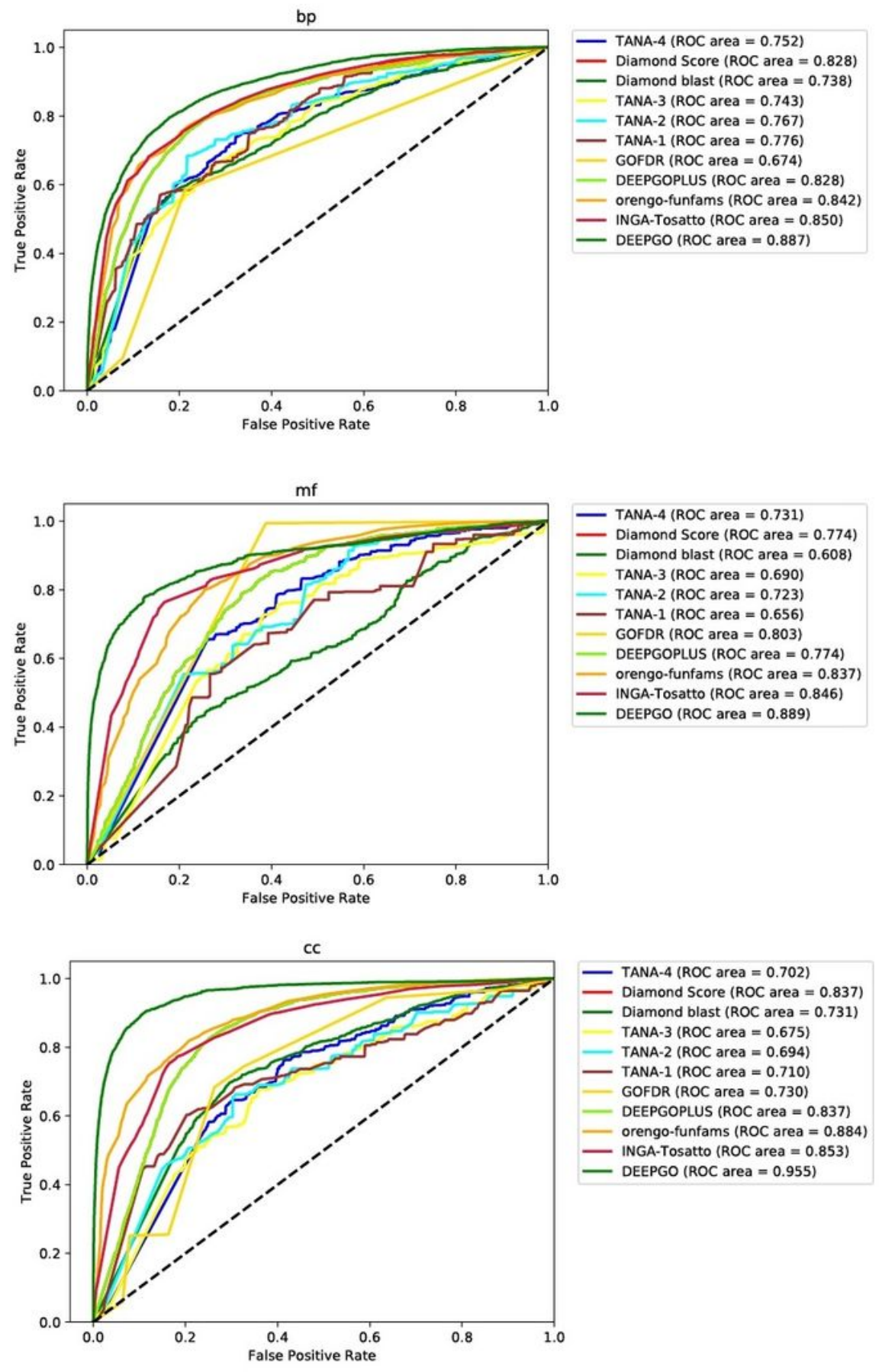

\section{Figure 1}

The ROC curves of TANA and other predictors over BP, MF and CC ontologies (All specie). 

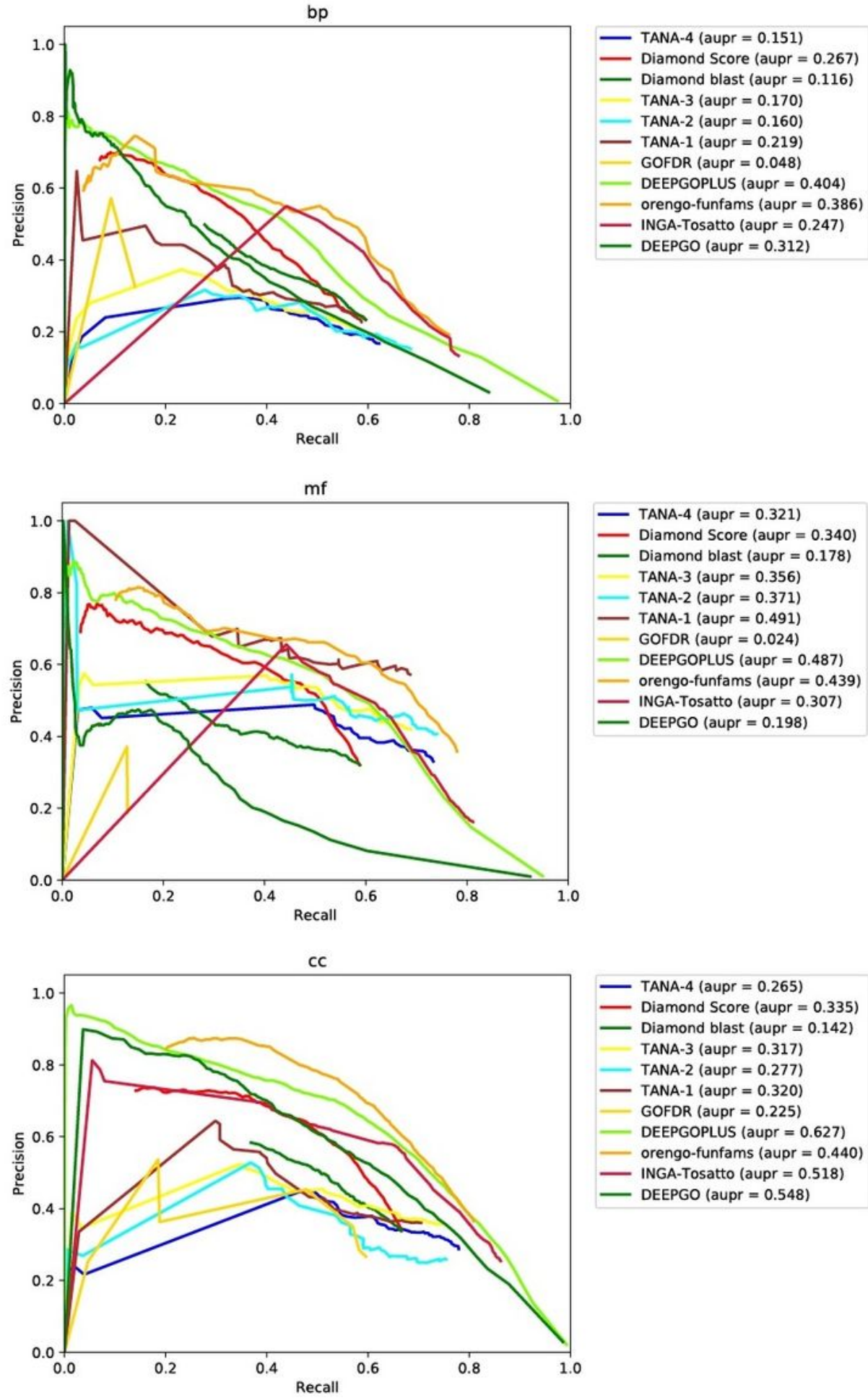

TANA-4 (aupr $=0.265$ )

Diamond Score (aupr $=0.335$ )

Diamond blast (aupr $=0.142$ )

TANA-3 (aupr $=0.317$ )

TANA-2 (aupr $=0.277)$

TANA-1 (aupr $=0.320$ )

GOFDR (aupr $=0.225$ )

DEEPGOPLUS (aupr $=0.627$ )

orengo-funfams (aupr $=0.440$ )

INGA-Tosatto (aupr $=0.518$ )

DEEPGO (aupr $=0.548$ )

\section{Figure 2}

The precision-recall plot of TANA and other predictors over BP, MF and CC ontologies (All specie). 


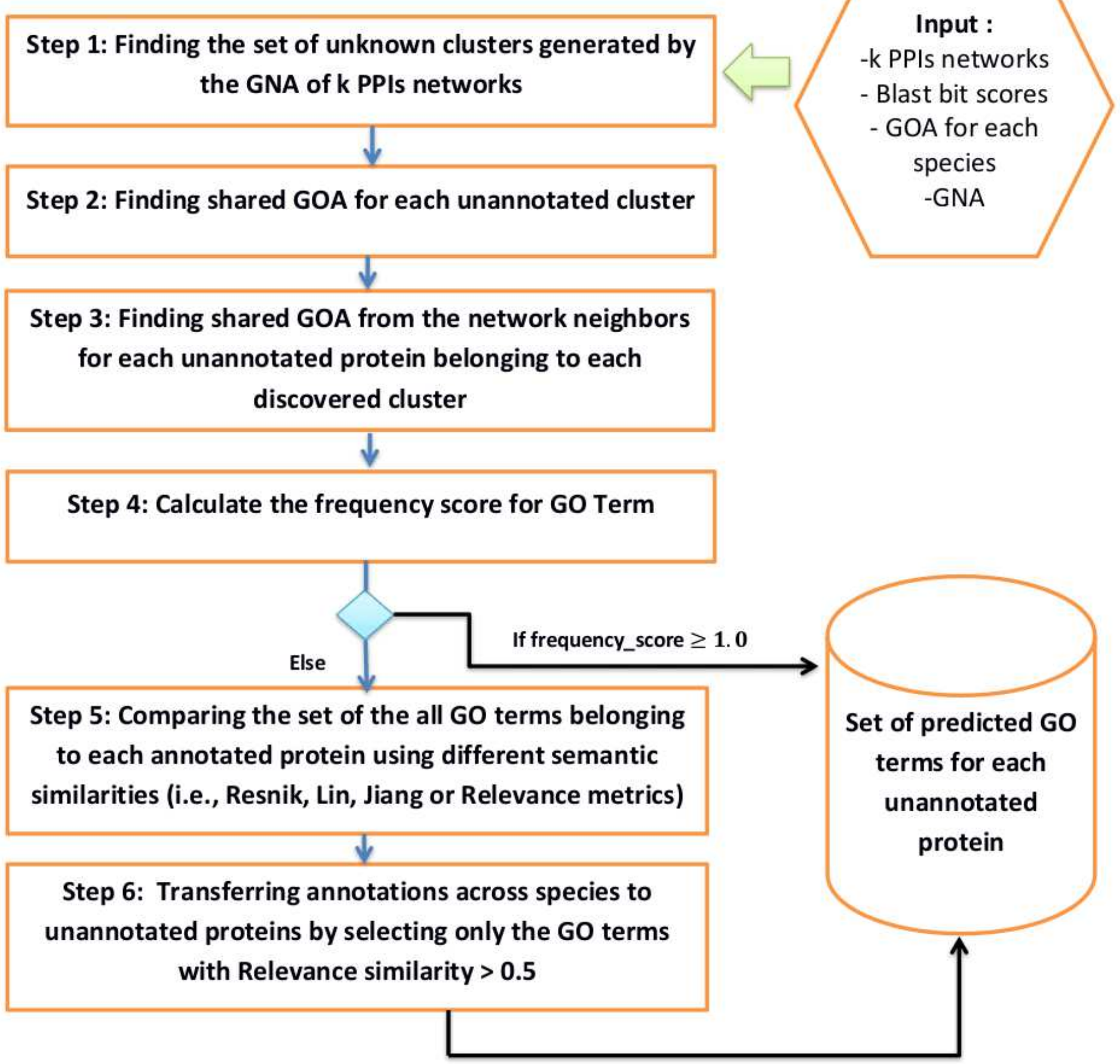

Figure 3

The different steps of TANA for scoring the prediction of GO terms for each unannotated protein. 


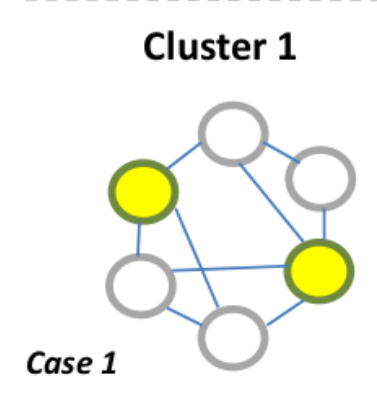

1 shared function

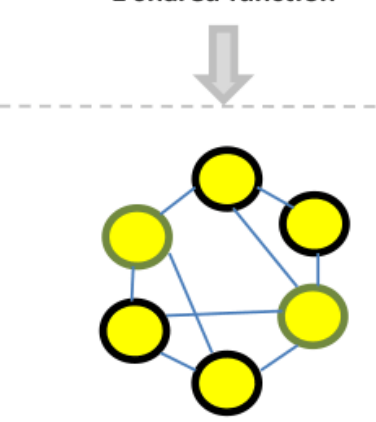

\section{Cluster 2}

Case 2

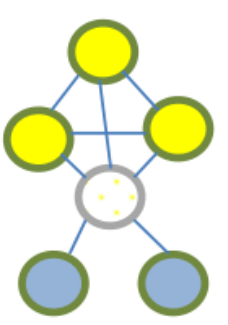

2 shared functions

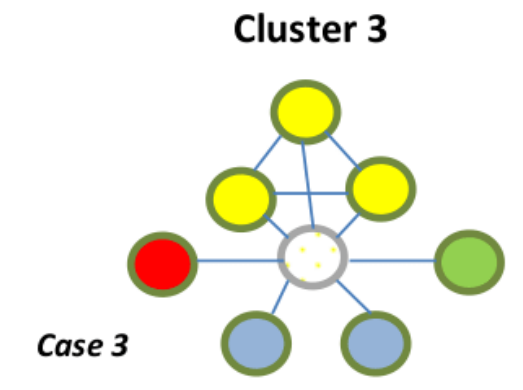

2 shared functions +2 consistent functions
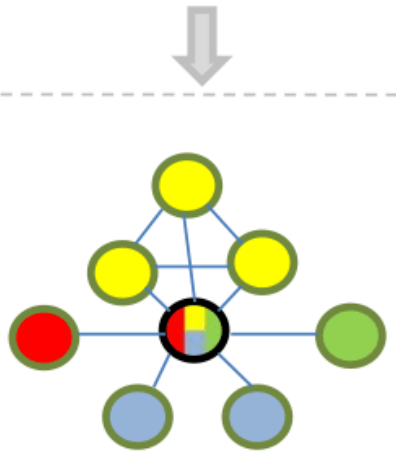

Note:

Unannotated protein

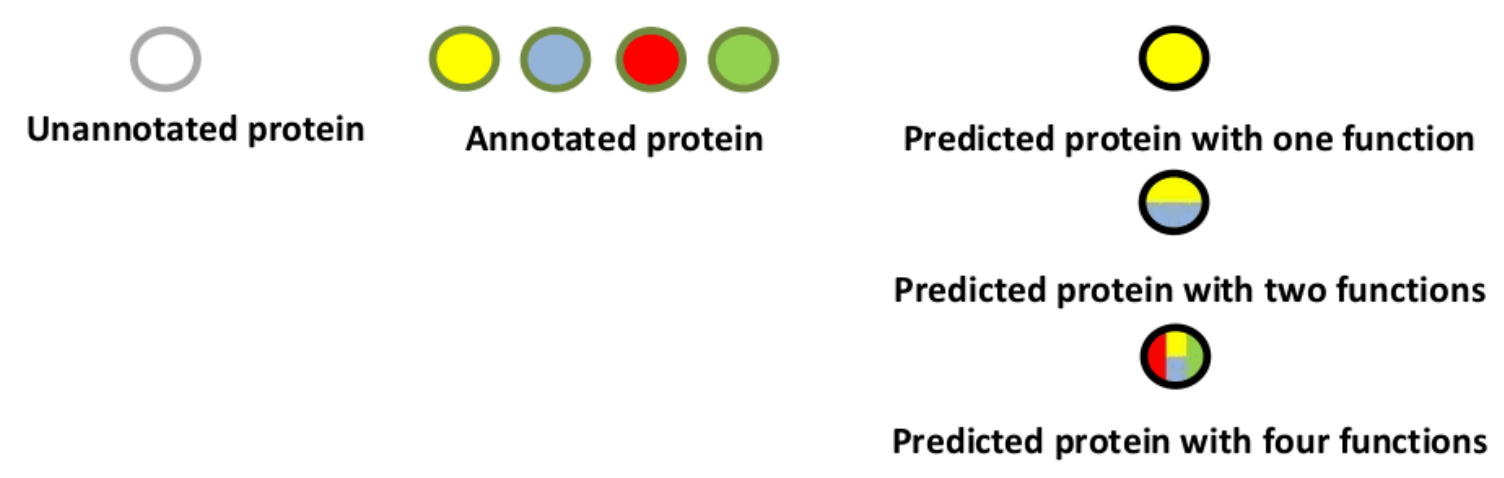

Annotated protein
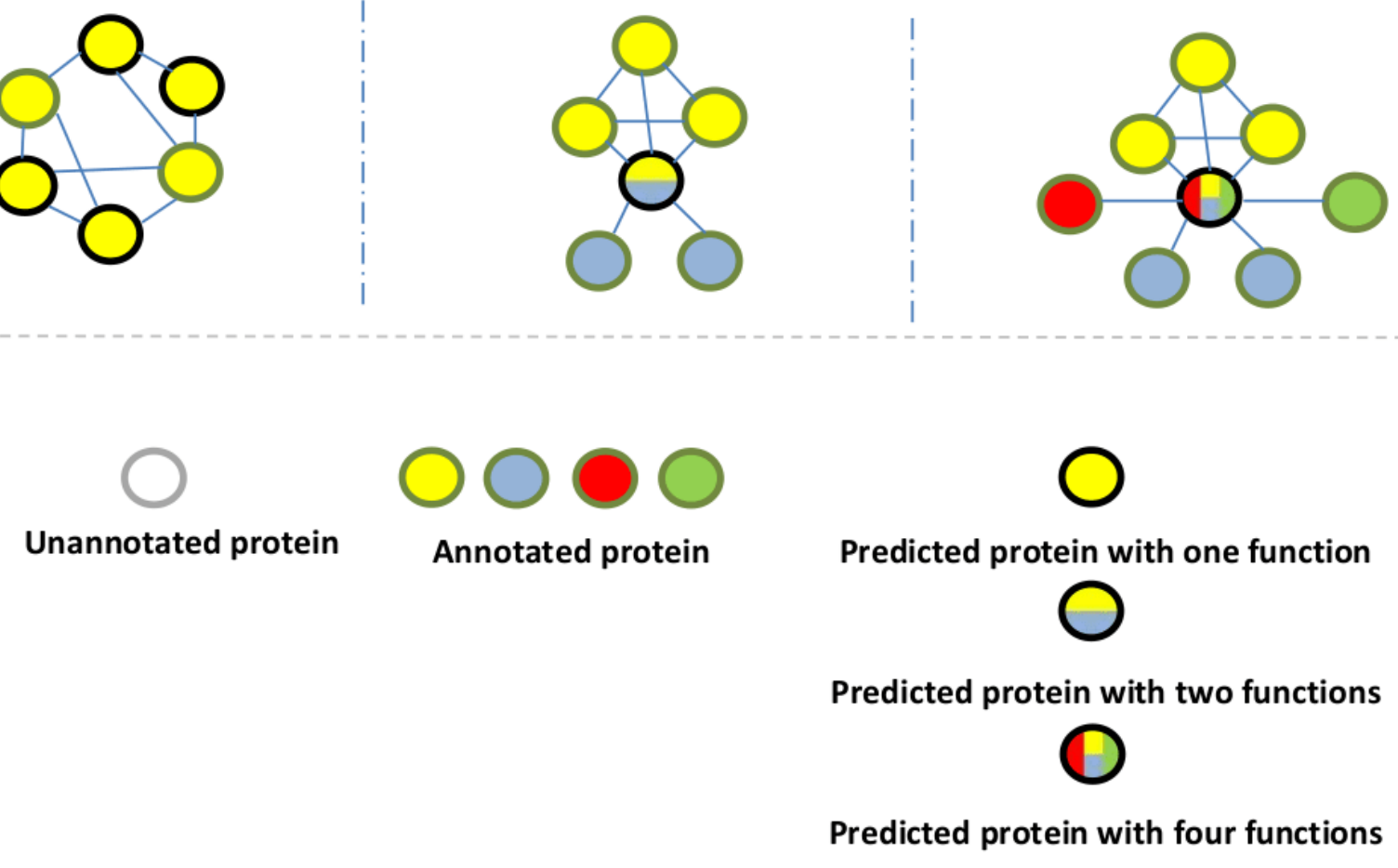

Predicted protein with four functions

Figure 4

Functional annotation yield by TANA. This shows clusters of proteins composed from unannotated proteins as well as annotated ones 


\section{Strategy 2: Transferring annotation from the GNA}

\section{Strategy 1: Transferring annotation from the "Direct neighborhood"}
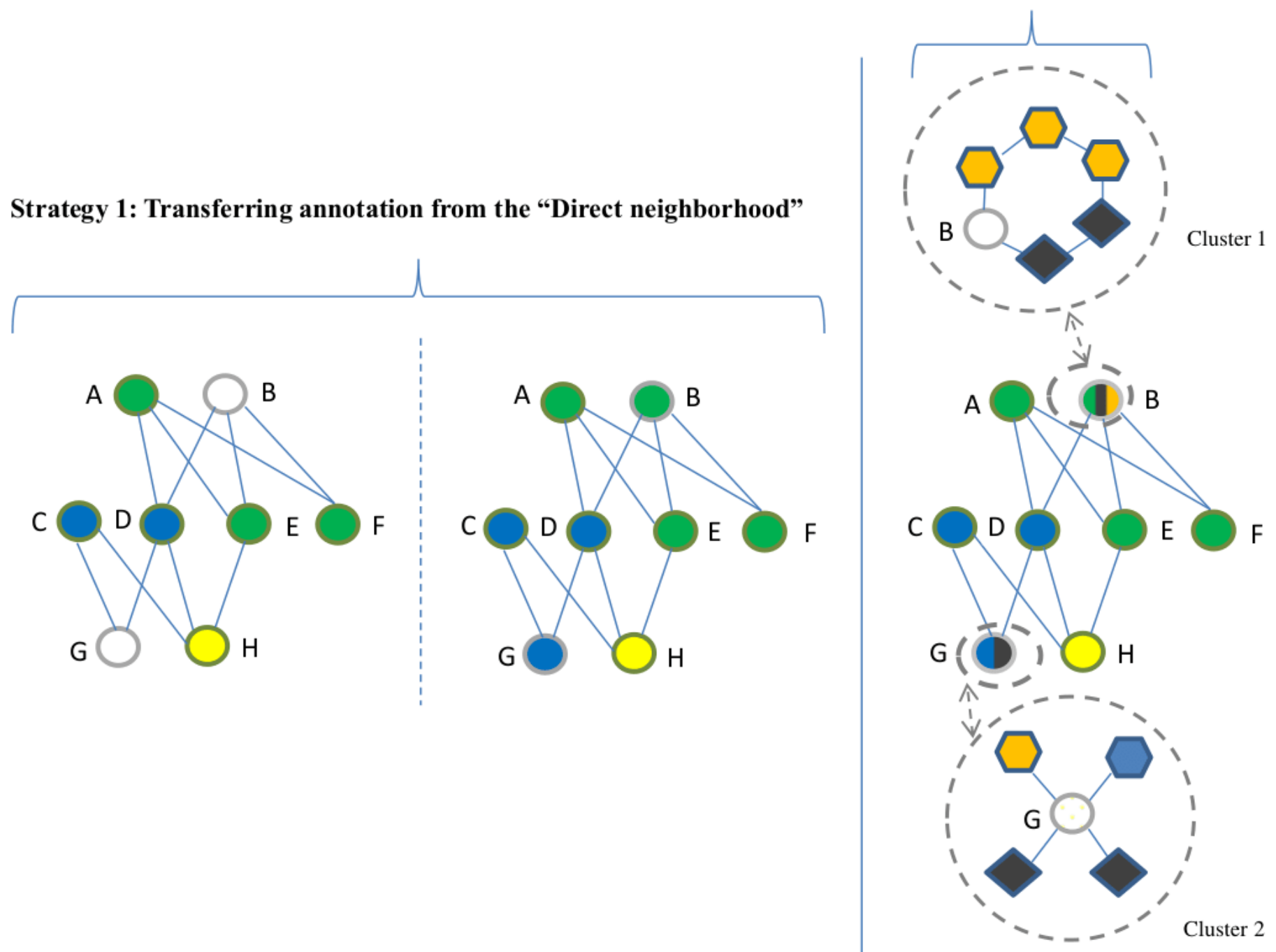

\section{Figure 5}

Two different strategies of protein function prediction are yield by TANA. In the PPI network on the left, proteins $A, E$, and $F$ are annotated with "green " function, proteins $C$ and $D$ are annotated with "blue" function. The node $\mathrm{H}$ is annotated with the "yellow" function and $\mathrm{G}$ and $\mathrm{B}$ are unannotated proteins (as denoted by white node color). With the "direct neighborhood" approach (the middle panel), node B gets annotated with "green" function, since the majority of its neighbors (E and F) have the "green" function. Similarly, $G$ gets annotated with "blue" function, since all of its neighbors ( $C$ and $D$ ) have the "blue" function. Adding to that, by using the other strategy based on transferring annotation from the alignment of PPI network, the protein B is also annotated with two shared functions (i.e, orange and black colors) coming from the "Cluster 1". Moreover, protein $\mathrm{G}$ also gets annotated with one shared function (i.e., Black color) from the shared function of the "Cluster 2". It is worth mentioning that: a) the proteins with a shape different from the circle node are a proteins coming from different species; $b$ ) The proteins $B$ and $G$ are 
the proteins with limited Knowledge, which had been experimentally annotated in one or two GO ontologies (BP, MF or CC).

\section{Supplementary Files}

This is a list of supplementary files associated with this preprint. Click to download.

- additionalfile1.1.pdf

- additionalfile1.2.pdf

- additionalfile1.3.pdf

- additionalfile1.4.pdf

- additionalfile1.5.pdf

- additionalfile1.6.pdf

- additionalfile1.7.pdf 